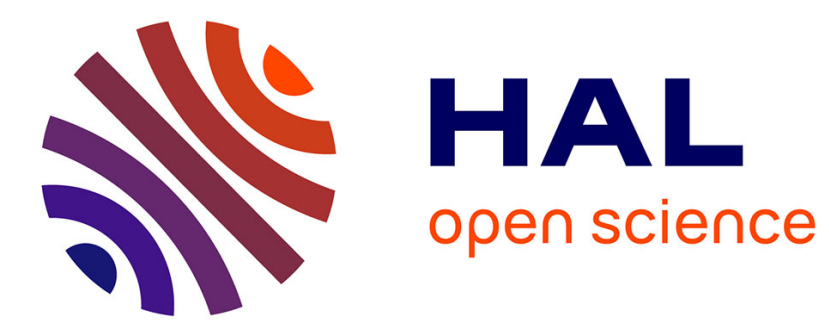

\title{
Set-Membership Estimation Improvement Applying HOSM Differentiators
}

\author{
Denis Efimov, Leonid Fridman, Tarek Raissi, Ali Zolghadri
}

\section{To cite this version:}

Denis Efimov, Leonid Fridman, Tarek Raissi, Ali Zolghadri. Set-Membership Estimation Improvement Applying HOSM Differentiators. ACC 2012, Jun 2012, Montreal, Canada. hal-00678978

\section{HAL Id: hal-00678978 \\ https://hal.inria.fr/hal-00678978}

Submitted on 14 Mar 2012

HAL is a multi-disciplinary open access archive for the deposit and dissemination of scientific research documents, whether they are published or not. The documents may come from teaching and research institutions in France or abroad, or from public or private research centers.
L'archive ouverte pluridisciplinaire HAL, est destinée au dépôt et à la diffusion de documents scientifiques de niveau recherche, publiés ou non, émanant des établissements d'enseignement et de recherche français ou étrangers, des laboratoires publics ou privés. 


\title{
Set-Membership Estimation Improvement Applying HOSM Differentiators
}

\author{
Denis Efimov, Leonid Fridman, Tarek Raïssi, Ali Zolghadri
}

\begin{abstract}
This work is devoted to design of interval observers for a class of Linear-Parameter-Varying (LPV) systems. Applying High Order Sliding Mode (HOSM) techniques it is possible to decrease the initial level of uncertainty in the system, which leads to improvement of set-membership estimates generated by an interval observer. In addition, it is shown that HOSM techniques may relax the applicability conditions of the interval observer design methods. The efficiency of the proposed approach is demonstrated through computer simulations.
\end{abstract}

\section{INTRODUCTION}

The problem of state estimation for nonlinear systems is very challenging and application important [4], [10], [18]. A complete palette of solutions exists for linear systems. In the nonlinear case, the most solutions are based on representation of the estimated system in a canonical form (frequently, partially linear), then particular approaches are available. In general case the LPV equivalent representation of nonlinear systems was found useful [15], [22], [25]. The basic idea is to replace the nonlinear complexity of the original system by an enlarged parametric variation in the LPV representation, which may simplify the observer design. There are several approaches to design observers for LPV systems [3], [11], [12], [17]. The present paper belongs to the framework of interval observers [3], [17]. That approach has been recently extended in [21] to nonlinear systems using LPV representations with known minorant and majorant matrices, and in [20] for observable nonlinear systems relaxing requirement on cooperativity (monotonicity) of the original system dynamics. The interval observers propagate the parameter uncertainty in the length of interval of the state estimation. The length of interval determines the estimation accuracy of the approach. This is why the uncertainty decreasing is very important for improvement of the interval (set-membership) estimation performance, which is the goal of the present work.

The HOSM techniques are very popular for design of observers for linear and nonlinear systems [1], [2], [5], [7],

The first and the last two authors are with University of Bordeaux, IMSlab, Automatic control group, 351 cours de la libération, 33405 Talence, France, \{Denis.Efimov; Tarek.Ra1ssi; Ali.Zolghadri\}@ims-bordeaux.fr (the present place of work of the first author is the Non-A project at INRIA LNE, Parc Scientifique de la Haute Borne, 40 avenue Halley, Bât.A Park Plaza, 59650 Villeneuve d'Ascq, France; and the present place of work of the third author is Conservatoire National des Arts et Métiers (CNAM), Département EASY, Cedric - laetitia 292, Rue St-Martin, case 2D2P10, 75141 Paris Cedex 03). The second author is with Dept. de Ingeniería de Control y Robótica, Facultad de Ingeniería UNAM, Edificio "Bernardo Quintana", Ciudad Universitaria D.F., México, 1fridman@servidor.unam.mx.
[23]. The sliding modes ensure a finite time of estimation error convergence to zero and complete insensitivity to a matched uncertainty [6], [16], [19]. Mainly these advances can be achieved under assumption that the systems is strongly observable or strongly detectable [2].

The objective of this work is to combine both approaches (the interval observers and the HOSM techniques) in order to improve accuracy of estimation achieved by the interval observers. Under a transformation of coordinates, an LPV system has a strongly observable subsystem. Applying HOSM differentiation approach it is possible to estimate the state and the state derivative for this subsystem, which can be further used for improved evaluation of the input and the parameter uncertainty in the rest part of the system. This combination improves the accuracy of the interval estimation. Additionally a relaxation of some applicability constraints usual for interval estimation can be obtained.

The paper is organized as follows. The system of interest, the basic facts from the theories of LPV systems, interval estimation and HOSM techniques are given in Section 2. The main result is described in Section 3. An example of computer simulation is presented in Section 4.

\section{PRELIMINARIES}

Euclidean norm for a vector $x \in \mathbb{R}^{n}$ will be denoted as $|x|$, and for a measurable and locally essentially bounded input $u: \mathbb{R}_{+} \rightarrow \mathbb{R}\left(\mathbb{R}_{+}=\{\tau \in \mathbb{R}: \tau \geq 0\}\right)$ the symbol $\|u\|_{\left[t_{0}, t_{1}\right]}$ denotes its $L_{\infty}$ norm:

$$
\|u\|_{\left[t_{0}, t_{1}\right]}=\operatorname{ess} \sup \left\{|u(t)|, t \in\left[t_{0}, t_{1}\right]\right\},
$$

if $t_{1}=+\infty$ then we will simply write $\|u\|$. Denote by $\mathcal{L}_{\infty}$ the set of all inputs $u$ satisfying $\|u\|<\infty$, and the sequence of integers $1, \ldots, k$ by $\overline{1, k}$.

In this work we consider the following LPV representation of a nonlinear system:

$$
\begin{gathered}
\dot{x}=A(\theta(t)) x+B(\theta(t)) u(t), \\
y=C x, \psi(t)=y+v(t),
\end{gathered}
$$

where $x \in \mathbb{R}^{n}, u \in \mathbb{R}^{m}, y \in \mathbb{R}^{p}, v \in \mathbb{R}^{p}$ are the state, the input, the output and the measurement noise of the system (1), $\psi(t)$ is the signal available for on-line measurements; $\theta \in \Theta \subset \mathbb{R}^{q}$ is the scheduling parameter vector, the set $\Theta$ is known; the matrix functions $A: \Theta \rightarrow \mathbb{R}^{n \times n}$ and $B: \Theta \rightarrow \mathbb{R}^{n \times m}$ are given. The instant values of $u(t) \in \mathcal{L}_{\infty}$, $v(t) \in \mathcal{L}_{\infty}$ and $\theta(t) \in \mathcal{L}_{\infty}$ are not known. Almost all 
existent approaches assume that the vector $\theta$ is accessible for measurements, in the following this assumption is relaxed, and only the domain $\Theta$ is given.

Assumption 1. $\|x\| \leq X,\|u\| \leq U$ and $\|v\| \leq V$, the bounds $X>0, U>0$ and $V>0$ are given.

Boundedness of the state $x$ and the inputs $u, v$ is a standard assumption in the estimation theory. Under Assumption 1 the signal $\psi(t)$ is also bounded.

\section{A. HOSM differentiation}

Taking the $s$-th time differentiable output $y(t)$ of the system (1), its derivatives can be estimated by the HOSM differentiator [13], [14] based on the noisy measurements $\psi(t)$ :

$$
\begin{gathered}
\dot{q}_{0}=\nu_{0}, \nu_{0}=-\lambda_{0}\left|q_{0}-\psi(t)\right|^{s / s+1} \operatorname{sign}\left[q_{0}-\psi(t)\right]+q_{1} \\
\dot{q}_{i}=\nu_{i}, i=\overline{1, s-1} \\
\nu_{i}=-\lambda_{i}\left|q_{i}-\nu_{i-1}\right|^{s-i / s-i+1} \operatorname{sign}\left[q_{i}-\nu_{i-1}\right]+q_{i+1} \\
\dot{q}_{s}=-\lambda_{s} \operatorname{sign}\left[q_{s}-\nu_{s-1}\right]
\end{gathered}
$$

where $\lambda_{k}, k=\overline{0, s}$ are positive parameters to be tuned.

Theorem 1. [14] Let $y: \mathbb{R}_{+} \rightarrow \mathbb{R}$ be s-th times continuously differentiable and $v(t) \in \mathcal{L}_{\infty}$ in (1), then there exist $0 \leq T<$ $+\infty$ and some constants $\mu_{k}>0, k=\overline{0, s}$ (dependent on $\lambda_{k}$, $k=\overline{0, s}$ only) such that in (2) for all $t \geq T$ :

$$
\left|q_{k}(t)-y^{(k)}(t)\right| \leq \mu_{k}|| v \|^{\frac{s-k+1}{s+1}}, k=\overline{0, s} .
$$

In particular, this result means that if $v(t) \equiv 0$ for all $t \geq 0$, then the differentiator (2) ensures the exact estimation of derivatives in a finite time. Application of HOSM differentiators for unknown input estimation and compensation in linear systems has been studied in [2], an extension to nonlinear systems is presented in [9].

\section{B. Interval estimation}

For two vectors $x_{1}, x_{2} \in \mathbb{R}^{n}$ or matrices $A_{1}, A_{2} \in \mathbb{R}^{n \times n}$, the relations $x_{1} \leq x_{2}$ and $A_{1} \leq A_{2}$ are understood elementwise. Given a matrix $A \in \mathbb{R}^{m \times n}$ or a vector $x \in \mathbb{R}^{n}$, define $A^{+}=\max \{0, A\}, A^{-}=A^{+}-A$ or $x^{+}=\max \{0, x\}$, $x^{-}=x^{+}-x$ respectively.

Lemma 1. Let $x \in \mathbb{R}^{n}$ be a vector variable, $\underline{x} \leq x \leq \bar{x}$ for some $\underline{x}, \bar{x} \in \mathbb{R}^{n}$.

1) If $A \in \mathbb{R}^{m \times n}$ is a constant matrix, then

$$
A^{+} \underline{x}-A^{-} \bar{x} \leq A x \leq A^{+} \bar{x}-A^{-} \underline{x} .
$$

2) If $A \in \mathbb{R}^{m \times n}$ is a matrix variable, $\underline{A} \leq A \leq \bar{A}$ for some $\underline{A}, \bar{A} \in \mathbb{R}^{m \times n}$, then

$$
\begin{gathered}
\underline{A}^{+} \underline{x}^{+}-\bar{A}^{+} \underline{x}^{-}-\underline{A}^{-} \bar{x}^{+}+\bar{A}^{-} \bar{x}^{-} \leq A x \leq \\
\bar{A}^{+} \bar{x}^{+}-\underline{A}^{+} \bar{x}^{-}-\bar{A} \underline{x}^{+}+\underline{A}^{-} \underline{x}^{-} .
\end{gathered}
$$

A matrix $A \in \mathbb{R}^{n \times n}$ is called Hurwitz if all its eigenvalues have negative real parts, it is called Metzler if all its elements outside the main diagonal are not negative. Any solution of the linear system

$$
\dot{x}=A x+\omega(t), \omega: \mathbb{R}_{+} \rightarrow \mathbb{R}_{+}^{n},
$$

with $x \in \mathbb{R}^{n}$ and a Metzler matrix $A$, is elementwise nonnegative for all $t \geq 0$ provided that $x(0) \geq 0$ [24]. Such dynamical systems are called cooperative (monotone) [24].

\section{MAIN RESULT}

For brevity of presentation the case $p=1$ is considered only (the case of vector measurements can be treated similarly). We will need the following assumptions.

Assumption 2. For all $\theta \in \Theta$, there is an invertible matrix $S(\theta) \in \mathbb{R}^{n \times n}$ such that the system (1) can be represented as follows:

$$
\begin{gathered}
x=S(\theta)\left[\begin{array}{c}
z_{1} \\
z_{2}
\end{array}\right], y=c^{T} z_{1}, \\
\operatorname{dim}\left\{z_{1}\right\}=n_{1}, \operatorname{dim}\left\{z_{2}\right\}=n_{2}, n_{1}+n_{2}=n, \\
\dot{z}_{1}=A_{0} z_{1}+b_{0}\left[a_{11}(\theta)^{T} z_{1}+a_{12}(\theta)^{T} z_{2}+b_{1}(\theta)^{T} u\right], \\
\dot{z}_{2}=A_{21}(\theta) z_{1}+A_{22}(\theta) z_{2}+B_{2}(\theta) u,
\end{gathered}
$$

where

$$
\begin{gathered}
c=\left[\begin{array}{ll}
1 & 0 \ldots 0
\end{array}\right]^{T}, b_{0}=\left[\begin{array}{lll}
0 \ldots 0 & 1
\end{array}\right]^{T}, \\
A_{0}=\left[\begin{array}{ccc}
0 & 1 \ldots 0 & 0 \\
\vdots & \ddots & \vdots \\
0 & 0 \ldots 0 & 1 \\
0 & 0 \ldots 0 & 0
\end{array}\right]
\end{gathered}
$$

is a canonical representation, the vector functions $a_{11}(\theta)$, $a_{12}(\theta), b_{1}(\theta)$ and the matrix functions $A_{21}(\theta), A_{22}(\theta), B_{2}(\theta)$ have corresponding dimensions.

It is worth to stress that for $n_{1}=1$ this assumption is always true (at least the output coordinate can be chosen in the vector $z_{1}$ ).

Assumption 3. Let there exist a vector function $f(\theta) \in \mathbb{R}^{n_{2}}$ such that

$$
\begin{gathered}
{\left[A_{22}(\theta) z_{2}+B_{2}(\theta) u\right]-f(\theta)\left[a_{12}(\theta)^{T} z_{2}\right.} \\
\left.+b_{1}(\theta)^{T} u\right]=\Delta_{1} z_{2}+\Delta_{2}(\theta) u
\end{gathered}
$$

for some Hurwitz matrix $\Delta_{1} \in \mathbb{R}^{n_{2} \times n_{2}}$ and $\Delta_{2}: \Theta \rightarrow$ $\mathbb{R}^{n_{2} \times m}$.

Assumption 4. There exists a matrix $P \in \mathbb{R}^{n_{2} \times n_{2}}$ such that the matrix $D=P^{-1} \Delta_{1} P$ is Hurwitz and Metzler $(H \& M)$.

Assumption 2 states that there exists a transformation coordinates, which represents the system (1) as a pair of interconnected subsystems (5) and (6). The subsystem (5) is strongly observable since it has the canonical representation $c, A_{0}, b_{0}$ (the conditions of existence of such a transformation for linear time-invariant systems are analyzed in [2]). However, the system is not necessarily detectable (the dynamics 
of (1) could be non-minimum phase as in [23]) since there is no requirement on stability of the matrix function $A_{22}(\theta)$. This relaxation may be important for application of interval observer design method for estimation in uncertain nonminimum phase systems. Instead, Assumption 3 states that the matrix $\Delta_{1}=A_{22}(\theta)-f(\theta) a_{12}(\theta)^{\mathrm{T}}$ is Hurwitz (the matrix $A_{22}(\theta)$ can be stabilized by an output feedback, or the pair of matrices $\left(A_{22}(\theta), a_{12}(\theta)^{\mathrm{T}}\right)$ is observable for all $\left.\theta \in \Theta\right)$ and independent in $\theta$. Under mild conditions of the main result in [20], in this case there is a matrix $P \in \mathbb{R}^{n_{2} \times n_{2}}$ such that $D$ is $\mathrm{H} \& \mathrm{M}$, as it is stated in Assumption 4.

Under these assumptions it is proposed to use the differentiator (2) to estimate the state $z_{1}$ and its derivative $\dot{z}_{1}$, then from (5) we get an improved estimate on the signal $a_{12}(\theta)^{\mathrm{T}} z_{2}+b_{1}(\theta)^{\mathrm{T}} u$, which can be applied for design of an interval observer for the system (6) in the new coordinates $r=P^{-1} z_{2}$. Let us consider these steps consequently.

Under Assumption 2 the output $y$ of the system (5) has $n_{1}$ derivatives. Therefore according to Theorem 1 and Assumption 1, there exist parameters $\lambda_{k}, k=\overline{0, n_{1}}$ in (2) with $s=n_{1}$ and $T>0$ such that for all $t \geq T$ :

$$
\left|q_{k}(t)-y^{(k)}(t)\right| \leq \mu_{k} V^{\frac{n_{1}-k+1}{n_{1}+1}}, k=\overline{0, n_{1}}
$$

for some constant $\mu_{k}, k=\overline{0, n_{1}}$. Thus $z_{1}(t)=\hat{z}_{1}(t)+$ $e_{1}(t)$ and $\dot{z}_{1, n_{1}}(t)=q_{n_{1}}(t)+e_{2}(t)$ for all $t \geq T$, where $\hat{z}_{1, i}(t)=q_{i-1}(t)$ and $\left|e_{1, i}(t)\right| \leq \mu_{i-1} V^{\frac{n_{1}-i+2}{n_{1}+1}}$ for $i=\overline{1, n_{1}}$, $\left|e_{2}(t)\right| \leq \mu_{n_{1}} V^{\frac{1}{n_{1}+1}}$. The variables $\hat{z}_{1}$ and $q_{n_{1}}$ are available for a designer, the errors $e_{1}$ and $e_{2}$ are upper bounded by some functions of $V$. Substitution of these variables into the last equation of (5) gives:

$$
q_{n_{1}}+e_{2}=a_{11}(\theta)^{\mathrm{T}}\left[\hat{z}_{1}+e_{1}\right]+a_{12}(\theta)^{\mathrm{T}} z_{2}+b_{1}(\theta)^{\mathrm{T}} u,
$$

or equivalently

$$
a_{12}(\theta)^{\mathrm{T}} z_{2}+b_{1}(\theta)^{\mathrm{T}} u=q_{n_{1}}+e_{2}-a_{11}(\theta)^{\mathrm{T}}\left[\hat{z}_{1}+e_{1}\right] .
$$

Substituting this equality in the differential equation (6) we obtain

$$
\begin{aligned}
\dot{z}_{2}= & \Delta_{1} z_{2}+\left[A_{21}(\theta)-f(\theta) a_{11}(\theta)^{\mathrm{T}}\right]\left(\hat{z}_{1}+e_{1}\right)+ \\
& f(\theta)\left(q_{n_{1}}+e_{2}\right)+\Delta_{2}(\theta) u
\end{aligned}
$$

which is a stable system according to Assumption 3.

Applying the transformation of coordinates $r=P^{-1} z_{2}$, the system (7) can be rewritten as follows

$$
\begin{aligned}
\dot{r}= & D r+G_{1}(\theta)\left(\hat{z}_{1}+e_{1}\right)+ \\
& G_{2}(\theta)\left(q_{n_{1}}+e_{2}\right)+G_{3}(\theta) u,
\end{aligned}
$$

where $G_{1}(\theta)=P^{-1}\left[A_{21}(\theta)-f(\theta) a_{11}(\theta)^{\mathrm{T}}\right], G_{2}(\theta)=$ $P^{-1} f(\theta)$ and $G_{3}(\theta)=P^{-1} \Delta_{2}(\theta)$. The dynamics of (8) is cooperative and stable, and all uncertain functions or variables in the right hand side of (8) belong to an interval for $\theta \in \Theta$ :

$$
\begin{gathered}
\underline{G_{j}} \leq G_{j}(\theta) \leq \overline{G_{j}}, j=\overline{1,3} ;|u(t)| \leq U ; \\
\left|e_{1, i}(t)\right| \leq \bar{e}_{1, i}=\mu_{i-1} V^{\frac{n_{1}-i+2}{n_{1}+1}}, i=\overline{1, n_{1}} ; \\
\left|e_{2}(t)\right| \leq \bar{e}_{2}=\mu_{n_{1}} V^{\frac{1}{n_{1}+1}}
\end{gathered}
$$

for all $t \geq T$, where the matrices $G_{j}, \overline{G_{j}}, j=\overline{1,3}$ are known. Therefore the following interval observer can be synthesized for (8):

$$
\begin{aligned}
& \dot{\bar{r}}=D \bar{r}+\left({\overline{G_{1}}}^{+}-{\overline{G_{1}}}^{-}\right) \hat{z}_{1}^{+}+\left({\underline{G_{1}}}^{-}-{\underline{G_{1}}}^{+}\right) \hat{z}_{1}^{-}+(9) \\
& \left({\overline{G_{1}}}^{+}+{\underline{G_{1}}}^{-}\right) \bar{e}_{1}+\left({\overline{G_{2}}}^{+}-{\overline{G_{2}}}^{-}\right) q_{n_{1}}^{+}+ \\
& \left({\underline{G_{2}}}^{-}-{\underline{G_{2}}}^{+}\right) q_{n_{1}}^{-}+\left({\overline{G_{2}}}^{+}+{\underline{G_{2}}}^{-}\right) \bar{e}_{2}+ \\
& \left({\overline{G_{3}}}^{+}+{\underline{G_{3}}}^{-}\right) U \text {, } \\
& \underline{\dot{r}}=D \underline{r}+\left({\underline{G_{1}}}^{+}-{\underline{G_{1}}}^{-}\right) \hat{z}_{1}^{+}+\left({\overline{G_{1}}}^{-}-{\overline{G_{1}}}^{+}\right) \hat{z}_{1}^{-} \\
& \left({\overline{G_{1}}}^{+}+{\underline{G_{1}}}^{-}\right) \bar{e}_{1}+\left({\underline{G_{2}}}^{+}-{\underline{G_{2}}}^{-}\right) q_{n_{1}}^{+}+ \\
& \left({\overline{G_{2}}}^{-}-{\overline{G_{2}}}^{+}\right) q_{n_{1}}^{-}-\left({\overline{G_{2}}}^{+}+{\underline{G_{2}}}^{-}\right) \bar{e}_{2}- \\
& \left({\overline{G_{3}}}^{+}+{\underline{G_{3}}}^{-}\right) U \text {, }
\end{aligned}
$$

the properties (3), (4) have been used to calculate (9), (10). Introducing the interval estimation errors $\bar{\epsilon}=\bar{r}-r, \underline{\epsilon}=r-\underline{r}$, we obtain

$$
\dot{\bar{\epsilon}}=D \bar{\epsilon}+\bar{\varepsilon}, \underline{\dot{\epsilon}}=D \underline{\epsilon}+\underline{\varepsilon},
$$

where $\bar{\varepsilon}=\left({\overline{G_{1}}}^{+}-{\overline{G_{1}}}^{-}\right) \hat{z}_{1}^{+}+\left({\underline{G_{1}}}^{-}-{\underline{G_{1}}}^{+}\right) \hat{z}_{1}^{-}+\left({\overline{G_{1}}}^{+}+\right.$ \left.${\underline{G_{1}}}^{-}\right) \bar{e}_{1}+\left({\overline{G_{2}}}^{+}-{\overline{G_{2}}}^{-}\right) q_{n_{1}}^{+}+\left({\underline{G_{2}}}^{-}-{\underline{G_{2}}}^{+}\right) q_{n_{1}}^{-}+\left({\overline{G_{2}}}^{+}+\right.$ $\left.G_{2}{ }^{-}\right) \bar{e}_{2}+\left(\bar{G}_{3}+G_{3}^{-}\right) U-G_{1}(\theta)\left(\hat{z}_{1}+e_{1}\right)-G_{2}\left(q_{n_{1}}+e_{2}\right)-$ $\overline{G_{3}}(\theta) u, \underline{\varepsilon}=G_{1}(\bar{\theta})\left(\hat{z}_{1}+e_{1}\right)+G_{2}\left(q_{n_{1}}+e_{2}\right)+G_{3}(\theta) u-$ $\left({\underline{G_{1}}}^{+}-{\underline{G_{1}}}^{-}\right) \hat{z}_{1}^{+}-\left({\overline{G_{1}}}^{-}-{\overline{G_{1}}}^{+}\right) \hat{z}_{1}^{-}+\left({\overline{G_{1}}}^{+}+{\underline{G_{1}}}^{-}\right) \bar{e}_{1}+$ $\left({\underline{G_{2}}}^{+}-{\underline{G_{2}}}^{-}\right) q_{n_{1}}^{+}+\left({\overline{G_{2}}}^{-}-{\overline{G_{2}}}^{+}\right) q_{n_{1}}^{-}-\left({\overline{G_{2}}}^{+}+{\overline{G_{2}}}^{-}\right) \bar{e}_{2}+$ $\left(\overline{G_{3}}+{\overline{G_{3}}}^{-}\right) U$. It is an arithmetic exercise to verify that under assumptions 1 and 2 (and the result of Theorem 1) the residual terms $\bar{\varepsilon}$ and $\underline{\varepsilon}$ are elementwise positive and bounded. Then using the results of monotone system theory [24] we prove that for all $t \geq T$

$$
\underline{r}(t) \leq r(t) \leq \bar{r}(t)
$$

and the estimates $\underline{r}(t), \bar{r}(t)$ are bounded, provided that

$$
\underline{r}(T) \leq r(T) \leq \bar{r}(T) .
$$

The former relation for the initial conditions can be easily satisfied since $\|x\| \leq X$ under Assumption 1. Using the property (3) we get for all $t \geq T$ :

$$
\begin{gathered}
\underline{z}_{2}(t) \leq z_{2}(t)=\operatorname{Pr}(t) \leq \bar{z}_{2}(t), \\
\underline{z}_{2}(t)=P^{+} \underline{r}(t)-P^{-} \bar{r}(t), \bar{z}_{2}(t)=P^{+} \bar{r}(t)-P^{-} \underline{r}(t) ; \\
\underline{z}_{1}(t) \leq z_{1}(t) \leq \bar{z}_{1}(t), \\
\underline{z}_{1}(t)=\hat{z}_{1}(t)-\bar{e}_{1}, \bar{z}_{1}(t)=\hat{z}_{1}(t)+\bar{e}_{1} .
\end{gathered}
$$

Defining $\underline{z}=\left[\underline{z}_{1}^{\mathrm{T}} \underline{z}_{2}^{\mathrm{T}}\right]^{\mathrm{T}}, \bar{z}=\left[\bar{z}_{1}^{\mathrm{T}} \bar{z}_{2}^{\mathrm{T}}\right]^{\mathrm{T}}$ and using (4) we finally formulate the interval estimates for the state $x$ :

$$
\begin{gathered}
\underline{S}^{+} \underline{z}^{+}-\bar{S}^{+} \underline{z}^{-}-\underline{S}^{-} \bar{z}^{+}+\bar{S}^{-} \bar{z}^{-} \leq x=S(\theta) z \leq \\
\bar{S}^{+} \bar{z}^{+}-\underline{S}^{+} \bar{z}^{-}-\bar{S}^{-} \underline{z}^{+}+\underline{S}^{-} \underline{z}^{-},
\end{gathered}
$$


which is satisfied for all $t \geq T$. Thus we have the following result.

Theorem 2. Let assumptions 1, 2, 3, 4 hold for the system (1). Then there exist the set of parameters $\lambda_{k}, k=\overline{0, n_{1}}$ in (2) and a constant $T>0$ such that for all $t \geq T$ the interval estimate (12) is true, provided that the condition (11) is satisfied for (9), (10).

Remark 1. The assumptions 3 and 4 can be replaced with the following one: there exists a vector function $f(\theta) \in \mathbb{R}^{n_{2}}$ such that

$$
\begin{gathered}
{\left[A_{22}(\theta) z_{2}+B_{2}(\theta) u\right]-f(\theta)\left[a_{12}(\theta)^{\mathrm{T}} z_{2}\right.} \\
\left.+b_{1}(\theta)^{\mathrm{T}} u\right]=\Delta_{1}(\theta) z_{2}+\Delta_{2}(\theta) u
\end{gathered}
$$

for some Hurwitz and Metzler matrix function $\Delta_{1}: \Theta \rightarrow$ $\mathbb{R}^{n_{2} \times n_{2}}$ and some $\Delta_{2}: \Theta \rightarrow \mathbb{R}^{n_{2} \times m}$. Next, the result of Theorem 2 can be obtained using the same technique and an interval observer from the paper [21].

\section{EXAMPLE}

To illustrate improvement of accuracy achieved in interval estimation by application of HOSM techniques consider a non-minimum phase system (the conventional techniques for the interval observer design [3], [17], [20] can not be applied in this case):

$$
\begin{aligned}
\dot{x}_{1} & =-a_{11}(\theta) x_{1}+a_{12}(\theta) x_{2}+b_{1}(\theta) u ; \\
\dot{x}_{2} & =a_{21}(\theta) x_{1}+a_{22}(\theta) x_{2}+b_{2}(\theta) u ; \\
y & =x_{1},
\end{aligned}
$$

where $x_{1} \in \mathbb{R}, x_{2} \in \mathbb{R}$ are the state variables, for all $\theta \in \Theta$

$$
\begin{gathered}
0.5 \leq a_{11}(\theta) \leq 1,-3 \leq a_{12}(\theta) \leq-1, \\
-0.5 \leq a_{21}(\theta) \leq 0.5,-0.5 \leq a_{22}(\theta)=0.5 a_{12}(\theta)+1 \leq 0.5, \\
0.5 \leq b_{1}(\theta) \leq 1,0.5 \leq b_{2}(\theta) \leq 1, U=1, V=0.1 .
\end{gathered}
$$

As we can see, the system (13) is already in the form (5), (6) with $x_{1}=z_{1}$ and $x_{2}=z_{2}$ (the matrix $S(\theta)$ equals to the identity, and Assumption 2 is satisfied). For simulation we use

$$
a_{11}(\theta)=0.75+0.25 \sin \left(x_{2} t\right), a_{12}(\theta)=-2+\sin \left(x_{1} t\right),
$$
$a_{21}(\theta)=0.5 \sin (t), b_{1}(\theta)=0.5, b_{2}(\theta)=0.75+0.25 \cos (0.5 t$

$$
\begin{gathered}
u(t)=U \sin (2 t), v(t)=V \sin (10 t), \\
\theta=\left[x_{1} x_{2} t\right]^{\mathrm{T}} .
\end{gathered}
$$

For the system (13) with the chosen parameters and the given input $u$ the state is bounded as follows $-3 \leq x_{2} \leq 1$ (Assumption 1 holds). It is easy to verify that for $f=0.5$ we have

$\left[a_{22}(\theta) z_{2}+b_{2}(\theta) u\right]-f\left[a_{12}(\theta) z_{2}+b_{1}(\theta) u\right]=\Delta_{1} z_{2}+\Delta_{2}(\theta) u$

for $\Delta_{1}=-1$ and $0 \leq \Delta_{2}(\theta) \leq 1.5$ (Assumption 3 is satisfied). Since $\Delta_{1}<0$ Assumption 4 is true with the matrix $P$ equals the identity.

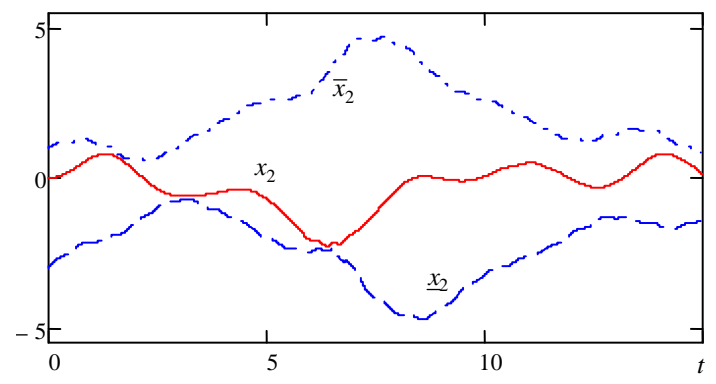

Figure 1. The results of simulation for non-minimum phase example

Therefore, according to Theorem 2 we may use the differentiator (2) to estimate $x_{1}$ and $\dot{x}_{1}$, that for $s=2$ can be reduced to the conventional super-twisting differentiator [13]:

$$
\begin{aligned}
& \dot{q}_{0}=-\lambda_{0} \sqrt{\left|q_{0}-\psi(t)\right|} \operatorname{sign}\left[q_{0}-\psi(t)\right]+q_{1} ; \\
& \dot{q}_{1}=-\lambda_{1} \operatorname{sign}\left[q_{0}-\psi(t)\right],
\end{aligned}
$$

where in our example $\lambda_{0}=20, \lambda_{1}=50, \hat{z}_{1}=q_{0}$ and $\bar{e}_{1}=|V|, \bar{e}_{2}=1.1 \sqrt{|V|}$. In this case the finite time $T=0.1$. Next, the interval observer (9), (10) generates the required set-membership estimates for the variable $x_{2}=r(-1 \leq$ $G_{1}(\theta)=P^{-1}\left[a_{21}(\theta)-f a_{11}(\theta)\right] \leq 1, G_{2}=0.5$ and $G_{3}(\theta)=$ $\left.\Delta_{2}(\theta)\right)$ :

$$
\begin{aligned}
& \dot{\bar{r}}=-\bar{r}+\hat{z}_{1}+2 \bar{e}_{1}+0.5 q_{1}+0.5 \bar{e}_{2}+1.5 U, \\
& \underline{\dot{r}}=-\underline{r}-\hat{z}_{1}-2 \bar{e}_{1}+0.5 q_{1}-0.5 \bar{e}_{2}-1.5 U .
\end{aligned}
$$

The results of this interval estimation are shown in Fig 1. It is worth to note that for the best knowledge of the authors, other existent approaches can not solve the problem of interval estimation for (13). In particular, application of a conventional interval observer design method [17], [21] is blocked by the non-minimum phase condition $\left(-0.5 \leq a_{22}(\theta) \leq 0.5\right)$. A further application of the sliding-mode estimation approaches [6], [16], [19] is blocked by the uncertainty presented in $\theta$.

\section{CONCLUSION}

The paper is devoted to application of the interval ob$t$ )servers and the HOSM differentiation to LPV system estimation. The HOSM techniques allow us to improve the estimation accuracy of an interval observer designed for LPV systems, or enlarge the class of LPV systems having an interval observer. The efficiency is shown on a uncertain and non-minimum phase example by computer simulations.

\section{REFERENCES}

[1] Barbot J.-P., Boutat D., Floquet T. An observation algorithm for nonlinear systems with unknown inputs. Automatica, 45, 2009, pp. 1970-1974.

[2] Bejarano F., Pisano A. Switched Observers for Switched Linear Systems with Unknown Inputs. IEEE Trans. Automatic Control, 56(3), 2011, pp. 681-686. 
[3] Bernard O., Gouzé J.L. Closed loop observers bundle for uncertain biotechnological models. J. Process Control, 14, 2004, pp. 765-774.

[4] Control and Observer Design for Nonlinear Finite and Infinite Dimensional Systems. Lecture Notes in Control and Information Sciences, Vol. 322, Meurer T., Graichen K., Gilles E.-D. (Eds), Springer, 2005.

[5] Moreno J.A., Osorio M. A Lyapunov approach to second-order sliding mode controllers and observers. Proc. 47th IEEE Conference on Decision and Control, 2008, pp. 2856-2861 .

[6] Edwards C., Spurgeon S.K. Sliding mode control: theory and applications. Taylor \& Francis, 1998.

[7] Efimov D., Fridman L. Global sliding-mode observer with adjusted gains for locally Lipschitz systems. Automatica, 47(3), 2011, pp. 565-570.

[8] Efimov D.V., Raïssi T., Zolghadri A. Stabilization of nonlinear uncertain systems based on interval observers. Proc. IEEE CDC-ECC 2011, Orlando, FL, 2011.

[9] Efimov D., Zolghadri A., Raïssi T. Actuators fault detection and compensation under feedback control. Automatica, 47, 2011, pp. 1699-1705.

[10] New Directions in Nonlinear Observer Design. Fossen T.I., Nijmeijer H. (eds.), Springer, 1999.

[11] Jaulin L. Nonlinear bounded-error state estimation of continuous time systems. Automatica, 38(2), 2002, pp. 1079-1082.

[12] Kieffer M., Walter E. Guaranteed nonlinear state estimator for cooperative systems. Numerical Algorithms, 37, 2004, pp. 187-198.

[13] Levant A. Robust exact differentiation via sliding mode technique. Automatica, 34(3), 1998, pp. 379-384.

[14] Levant A. High-order sliding modes: differentiation and outputfeedback control. Int. J. Control, 76(9-10), 2003, pp. 924-941.

[15] Marcos A., Balas J. Development of linear-parameter-varying models for aircraft. J. Guidance, Control, Dynamics, 27(2), 2004.

[16] Modern Sliding Mode Control Theory: New Perspectives and Applications. Lecture Notes in Control and Information Sciences, Vol. 375, Bartolini G., Fridman L., Pisano A., Usai E. (Eds.), Springer, 2008.

[17] Moisan M., Bernard O., Gouzé J.L. Near optimal interval observers bundle for uncertain bio-reactors. Automatica, 45(1), 2009, pp. 291-295.

[18] Nonlinear Observers and Applications. Lecture Notes in Control and Information Sciences, Vol. 363, Besançon G. (Ed.), Springer, 2007.

[19] Sliding mode control in engineering. Perruquetti W., Barbot J.P. (Eds.), Marcel Dekker, 2002.

[20] Raïssi T., Efimov D., Zolghadri A. Interval state estimation for a class of nonlinear systems. IEEE Trans. Automatic Control, 57(1), 2012, pp. 260-265.

[21] Raïssi T., Videau G., Zolghadri A. Interval observers design for consistency checks of nonlinear continuous-time systems. Automatica, 46(3), 2010, pp. 518-527.

[22] Shamma J., Cloutier J. Gain-scheduled missile autopilot design using linear parameter-varying transformations. J. Guidance, Control, Dynamics, 16(2), 1993, pp. 256-261.

[23] Shtessel Yu.B., Baev S., Edwards C., Spurgeon S. HOSM Observer for a Class of Non-Minimum Phase Causal Nonlinear MIMO Systems. IEEE Trans. Automatic Control, 55(2), 2010, pp 543-548.

[24] Smith H.L. Monotone Dynamical Systems: An Introduction to the Theory of Competitive and Cooperative Systems, vol. 41 of Surveys and Monographs, AMS, Providence, 1995.

[25] Tan W. Applications of Linear Parameter-Varying Control Theory. PhD thesis, Dept. of Mechanical Engineering, University of California at Berkeley, 1997. 